Copyright 1979 Optical Society of America

\title{
Solitons created by chirped initial profiles in coherent pulse propagation
}

Hmurcik, Lawrence V.; Kaup, D. J.

Journal of the Optical Society of America

L.V.Hmurcik and D.J.Kaup, "Solitons created by chirped initial profiles in coherent pulse propagation", Journal of the Optical Society of America, v69, p 597 (1979) 


\title{
Solitons created by chirped initial profiles in coherent pulse propagation
}

\author{
L. V. Hmurcik and D, J, Kaup \\ Department of Physics, Clarkson College of Technology. Potsdam, New York 13676 \\ (Receved 23 May 1978, revised 2 October 1978 )
}

\begin{abstract}
If an incident pilse is chiped, the critical parameters for selfinduced transparency to occlin in coherent pulse propagation can ro longer be obtained from the well knowh MicGallHahn area theo rem. We have been able to obtain these paraneters by solving the Zakharovs Shabat eigenvalue equation for the bound state eigenvalues. We find that critical (threshold) areas will be increased for a chipped incident pulse in almost all cases, except for a box profile or for a pulse that is approximately box-1ike in shape. In these latter cases, the chirped critical areas will instead decrease for the second and all higher branches. The first branch's critical area is always increased due to chirping:
\end{abstract}

\section{NTROBUCTION}

The phenomenon of self induced transparency (SIT) was first treated theoretically by McCalland I Lhn' 2 in 1967 and they also presented experimental evidence for the existence of SIT in ruby. As they had shown, SIT is a nonlinear phenomenon which will hot occur unless the initial pulse is suf ficiently intense. When the initial pulse profile is unehirped, monotonic and has only one extremun, they were able to show, from their area theorem, that SIT could only occur if the area of pulse profile exceeded $\pi$. Of course, if the area of the initial pulse profile were zero, but the pulse still nontrivial, then their area theorem gave a trivial result, which could not determine the critical conditions for SIT to occur. Also, when the nintial profile is chirped, the McCall Hahn area theorem is no longer valid, so again, the exact critical conditions for SIT to occur are not krown. The off resonance case was investigated numerically by Diels and Hahn ${ }^{3}$ in 1973. Their results indicated that the off-resonance critical area might be possibly less than $\pi$ for certain pulses. However, based on the resuits of Lamb, such is not the case, and instead, the exact critieal area does remain at $\pi$

In this paper, we shall present the results of our numerical investigations on chirped nitial profiles, and shall compare and contrast the chirped and unchirped cases in reference to critical absolute areas. form

To start with, consider an electromagnetic pulse of the

$$
E(x, t)=\delta(x, t) \cos (k x-\omega t+\delta)
$$

where $\mathcal{G}$ is the real, slowly varying envelope of the puise. We shall find it convenient to use instead the complex notation where

$$
E=\operatorname{Re}\left[(x, t) e^{i(x)-\omega)}\right],
$$

and where 6 is the complex electric field envelope, given by. $=6 e^{28}$

As is well known, in SIT an unchirped pulse incident on a medium is resthaped into a number of $2 \pi$ pulses which prop agate losslessly, while the remaining pulse energy is absorbed by the medium within a few Beer's lengths. (In the inverse scattering solution of this problem, 5,6 the $2 \pi$ pulses'are the solitons, while the pulse energy which is eventually absorbed in the "radiation," which usually dies out exponentially) From the McCall Hahn area theorem, , 2 the number of emerging $2 \pi$ pulses can be predicted for simple unchirped profiles: In this case, $\in$ nust be real, and the critical areas are found to be odd integral multiples of $x$. Slusher and Gibbs? later experimentally verified many of the theoretical results of Mccall and Hahn.

But the area theoren is valid only for the unchirped, on resonant case. For the chirped and/or off resonant cases, more powerful techniques are required for determining critical parameters. Noting that many of the properties of $2 \pi$ pulses were the same properties as found in KdV solitons, I L mb4, eventually was able to transform ${ }^{\circ}$ the Bloch equations into the well known Zakharov Shabat eigenvalue problen, 1 which is used in the "inverse scattering transform" "A? Although he could not solve the general case (since he had not yet incor porated the Maxwell equation, still he was able to deduce many features of the solution, handle off-resonant and chirped profiles, and in particular, 4 discuss the effects due to off res. onance. Shottly thereafter, Ablowitz, Kaup, and Newell6 were able to include the Maxwell equation fully into Lamb's result, thereby obtaining a complete solution. Most impor tantly, they could then show that the problen of determining how many $2 \pi$ pulses and their final pulse heights, was reduced to simply solving an eigenvalue problem (the Zakharoy Sha bat problem) for its bound states. This eigenvalue problem is 56

$$
\begin{aligned}
& v_{1}+l_{1} v_{1}=(1 / 2) k_{k} \in v_{2} \\
& v_{2},-i s v_{2}=(1 / 2)_{k \in} v_{2},
\end{aligned}
$$

where in $(3), v_{1,2}$ are the components of the eigenfunction, is the eigenvalue, $\mathrm{e}$ is given by (2), and $\%$ is the Mc Gall $\mathrm{Hahn}^{1 \text { i. }}$ ,6 with which the area is defined by

$$
\theta(x)=r \iint_{-}^{\infty} d t \delta(x, t)
$$

when $r=6$. The eigenvalue $S$ is in generat complex and is written $\}=s+i$. The variable $t$ in $(3)$ is simply the retarded tine, given by

$$
r=t-n+1 c
$$

where $n$ is the index of refraction, and for our second coord nate, ve shall use

$$
x=x
$$

Using (3), Lamb' and Ablowitz et al, 6 found that the 2 , pulses do correspond to refectionless potentials and that the 
bound state eigenvalues do determine the pulse parameters. For a single $2 \pi$ pulse, they found that 4,6

$$
\alpha \epsilon=4 \eta \exp [(e+b x-2 \xi)] / \cosh (f+d x-2 \eta \tau)
$$

where $e$ and $f$ are phase factors, and $b$ and $d$ are constants depending on the inhomogeneous broadening fbroadening due to natural line widths and Doppler broadening) In (6) we have the typical sech shape for a $2 \pi$ pulse. 1 The solt tor pulse $h$ ight and the inverse of the width are proportional to $\eta$ the inaginary part of 5 while the instantaneous soliton frequency (relative to the natural atomie frequency) is given by $2 \xi$, where $\zeta$ is the real part of $\zeta$. Iherefore, regardless of the medium and for any given incident pulse shape, we can find the exact size, frequeney and number of all $2 \pi$ pulses produced by simply finding the bound state eigenvalues of $(3)$

Inthispaper we shall numerically solve for these bound state eigenyalues tor three different nil al (chirped) pulse profiles first we shall describe how these calculations were made, and then we shall present our tesults

As is usually done in the inverse scattering method, 12 we consider the $\phi$ and $\psi$ solutions of (3) defined by

$$
\begin{aligned}
& \text { W }
\end{aligned}
$$

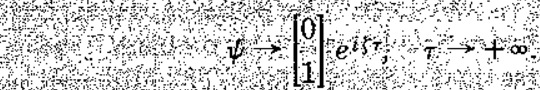

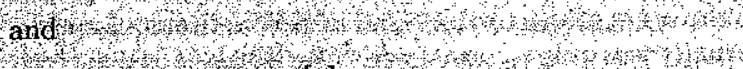

Now, as $\tau>+$, the $\phi$ solution becomes

$$
\begin{array}{ll}
W \\
y
\end{array}
$$

which defines $a(s)$ and $b(\xi)$, One also has 1,

$$
a-\omega(\phi)=\phi_{1} \psi_{2}-\phi_{2} \psi_{1}
$$

When is in the upper half complex plane from $(7)$ we see that bound states can only occur when $a(b)=0$, Thus by numercally calculating $a(y)$ we can determine the bound state eigenvalues by noting where $a(\xi)$ goes to zero, We cal culate $\alpha(9)$ by frrst using (3) and $(7$ a) to determine $\phi$ on the Left, then (3) and ( $7 \mathrm{~b})$ to determine $\psi$ on the right, and then evaluate a( $($ by (8), using the values of $\phi$ and $\psi$ at some common value of $t$ This is done for as many values of $y$ as is required. With the exeeption of the chirped box profle, Which can bo solvedin terms of parabolic cylinder functions of complex arguments, no other pulse shape exists to date for which we canobtain an analytic solytion of $(3)$, when the pulse is chirped:

Since our bound state solutions will be numerical it would be very advantageous if we could restrict our search for solutions to a particular portion of the complex $\xi$ plane, We would then have a limited range of $\zeta$ values to check when searching tor bocud state solutions, One way to do this was shown by Ablowitz et at 6 For oreal, bound-statesolutions were shown to he either on the positive $n$ axis $(2 \pi$ pulses) and $/ 0$ to occur as conjugate pairs, $n+\xi$ (breathers?), Now for complex but also symmetric in $\mathrm{\tau}$ we can show that this criterion still holds (See Appendix A) Thus by restricting our attention to symmetic chirped pulses, ovir search is greatly simplified, and we can fird the bound state eigenvalues much faster.
This procedure still leaves one possible difficulty. $/ \mathrm{h}$ searching for eigenvalues, how do we know when we have found all of them? This can be determined by looking at the phase of $a(\zeta)$ tor $\zeta$ real As discussed in Ref 12 , wave

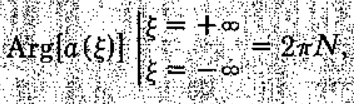

where N is the total number of zeros of a in the upper half plane. By finding $\mathrm{N}$ first, we then know exactly how many bound state eigenvalues exist.

In these investigations, welused only a simple chirp. Formally we can define a ehirp as

$$
\frac{1}{2} \partial, \gamma(x)
$$

Note, a purse with constant chinp is actually onlyofr reso nance and thenefore constant chins wil hol be considered here) The simplest hontrivid ehirp is one that varies linearly $1 \mathrm{t} t$ Hence $\delta$, 2 Thus ot $x=0$. from $(5)$, whave $\delta=$ $\Delta T_{t}^{2}$ where $\Delta$ is a dimensionless constan called the chirp strength, and to s the pulse width for targe, this chro factor oscillates rapidly which caused some numerical dif ficulties. Fos this reason at times we use the alternate form of

$$
\gamma=\Delta \beta(\tau / p) 2]\left[\beta+(, /,)^{2}\right]
$$

By taking $\beta$ large, our chiro would eventually become zero at very larve yalues of t but for 0 , $p$, the chit would stil be linear in $\tau$ h addition, we found that both forms of a chirp gave almost identled resuits for $\beta>1$, as expected. Thus we found that the behavior of the chirp factor at large r was rel atively unimportant, as one might expect

Once we had decided on an incident pulse shape $(0$, , and chirp factor $e^{i}$, we simply used the following four steps to gather our data- (1) First, we used (4) to parameterrze $\mid$ of in terms of the rnitial absolute area $\theta_{0}$ and a pulse width $D_{D}$. We then chose one particular value for $\theta_{0}$ and $\Delta$ (ii) We plotted a $(\zeta)$ for $\eta$ o from some arge negative 5 The overal phase change no then told us the number of bound of state solutions which existed (1i) We then found al the bound state solv tions which correspond to the zeros of $\alpha$ along the positiven axis. We could then plot these eigenvalues as functions of $\theta_{0}$ If there remained any zeros of $a(s)$ a fer scanning the inag nary axis, we would then search in the complex plane for the remaining breather states. (iv) Now we chose another value for $\theta_{0}$, and repeated steps (in) and (iii), etc

Let us note that there are at least two different definitions in the literature for the area of chirped pulses. Diels and Hahn took it to be the amplitude of the Fourier transform at the average frequency Deck and Lamb 3 implicitly chose it to be the integral of the absolute magnitude of the field The advantage of the first definition is that is an experi mentally measurable quantity The ad vantage of the second definition is that once the profile is fixed, it correlates directly with the initial pulse energy, $f \in t d t, w h c h$ is chirp independent. We have chosen the second defintion for conve nience and also so that our thresholds would correlate someWhat to the required energies of course given the initial profile and chirp, these two areas can be related. 


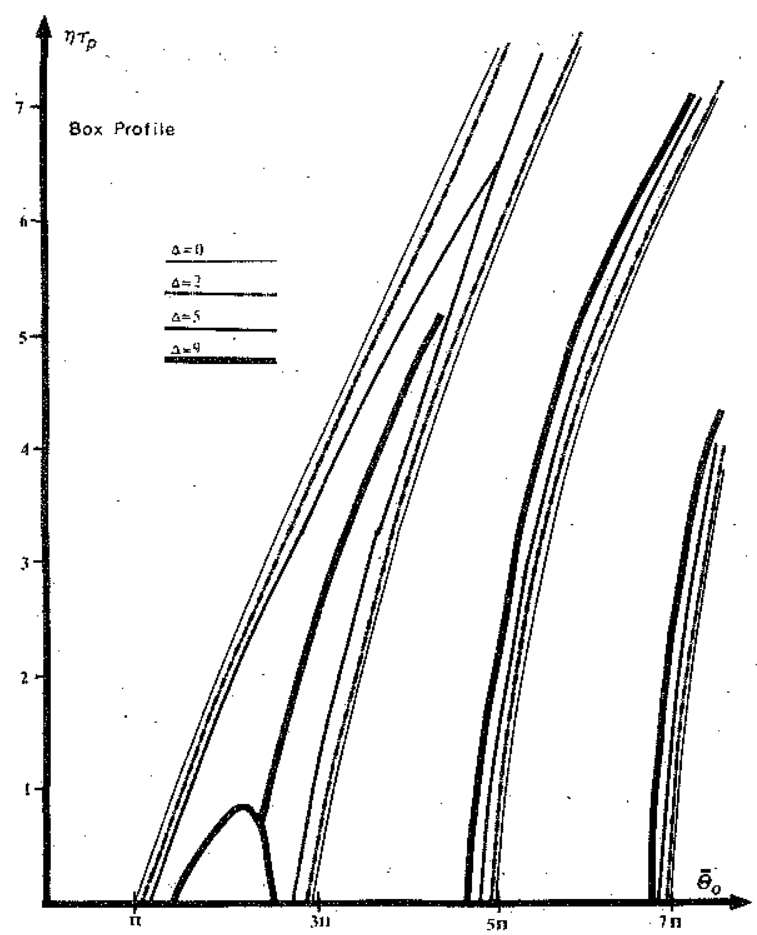

FIG. 1. Graph of $\eta \tau_{p}$ vs $\vec{\theta}_{0}$ for box profile with chirp strengths $\Delta=0,2$, 5,9 .

\section{EFFECTS OF CHIRPING ON THE BOX PROFILE}

For the first case of chirping, we chose $\kappa \mathscr{E}=\bar{\theta}_{0} / \tau_{p}$ if $|\tau|<$ $\tau_{p} / 2$ and $\mathscr{E}=0$ otherwise. Then since this profile has a finite extent, we took $\beta=\infty$ in (10), which gave the complex electric field envelope to be $\epsilon=\mathscr{E} \exp i \Delta\left(\tau / \tau_{p}\right)^{2}$. With this choice for $\epsilon$, we solved (3) numerically. After finding the bound-state eigenvalues, $(\zeta=i \eta)$ for various initial areas $\bar{\theta}_{0}$, we plotted $\eta \tau_{p}$ vs $\bar{\theta}_{0}$ in Fig. 1 for chirp strengths $\Delta=0,2,5,9$.

For $\Delta=0$ (unchirped) we obtain the same critical areas as obtained from the McCall-Hahn area theorem. (We define the critical area as the absolute area of the input pulse just large enough to produce one more additional soliton, usually of zero amplitude.) For $\Delta=2$, the second and all higher branches shift to lower areas with respect to the unchirped branches. However, for the first branch, the critical areas for the chirped cases are larger than for the unchirped case. Furthermore, for $\Delta=5$ all branches again shift as they did for $\Delta=2$, but now the first two branches merge together into one single branch. Both branches now have the same imaginary eigenvalue $\eta$ but they also have equal and opposite real nonzero values of $\xi$. Therefore, where these branches merge, our eigenvalues are occurring in conjugate pairs, $\zeta_{k}$ and $-\zeta_{k}^{*}$, and as we have already noted, these eigenvalues then correspond to a "breather" solution. If the inhomogeneous broadening is symmetric ${ }^{5}$ so that the velocity of the $\zeta_{k}$ soliton and the $-\zeta_{k}^{*}$ soliton are the same, then these solutions will indeed propagate as a breather. But, if these velocities would differ, then eventually, this "breather" would separate into two individual solitons, and no longer would it be multisoliton bound state. $^{11}$

Since the box profile is on compact support, it is also possible to check these numerical results with a perturbation analysis of (3) for $\Delta$ small. Note that at the critical areas, $\zeta$
$=0$. So we simply solve (3) with $\zeta=0$, and find what values of $\bar{\theta}_{0}$ gives $a=0$. As shown in Appendix B, one can obtain the solution of (3) at $\zeta=0$ as an expansion in $\Delta$, and we let $\vec{\theta}_{\text {cr }}$ (chirped) $=(2 n-1) \pi+\gamma_{n}$ where $(2 n-1) \pi$ are the unchirped critical areas. One finds that

$$
\gamma_{n}=\frac{\Delta^{2}}{\left[(2 n-1) \pi^{2}\right.}\left(\frac{2}{(2 n-1) \pi}-\frac{(2 n-1) \pi}{6}\right)+0\left(\Delta^{4}\right) .
$$

For the first branch $(n=1)$, (11) gives $\gamma_{1}>0$ so that chirped critical area does increase over the unchirped critical area, for the first branch. But for all other branches, $(n>1),(11)$ gives $\gamma_{n}<0$, so that chirping will cause the critical area to decrease.

We should also note that an almost identical problem has been solved by DuBois, Forslund, and Williams. ${ }^{14}$ However, in their work, they were concerned with instabilities in plasmas, and from the nature of their problem, they were interested in comparing (in our notation) $\eta \tau_{p} / \bar{\theta}_{0}$ vs $\left(\Delta \tau_{p}\right)^{1 / 2}$. Thus although their graphs are different from ours, they basically contain the same information.

\section{EFFECTS OF CHIRPING THE SECH AND GAUSSIAN PROFILES}

The results for the sech and Gaussian profiles are somewhat different than that for the box profile. These profiles are defined by

$$
\begin{gathered}
\kappa \mathscr{E}=\left(\bar{\theta}_{0} / \pi \tau_{p}\right) \operatorname{sech}\left(\tau / \tau_{p}\right), \\
\kappa \mathscr{E}=\left(\bar{\theta}_{0} / \sqrt{\pi} \tau_{p}\right) \exp \left[-\left(\tau / \tau_{p}\right)^{2}\right],
\end{gathered}
$$

where again $\bar{\theta}_{0}$ is the absolute area of $\kappa \mathscr{E}$. As we already have mentioned, since chirp factors of the form

$$
\exp i \Delta\left(\tau / \tau_{p}\right)^{2}
$$

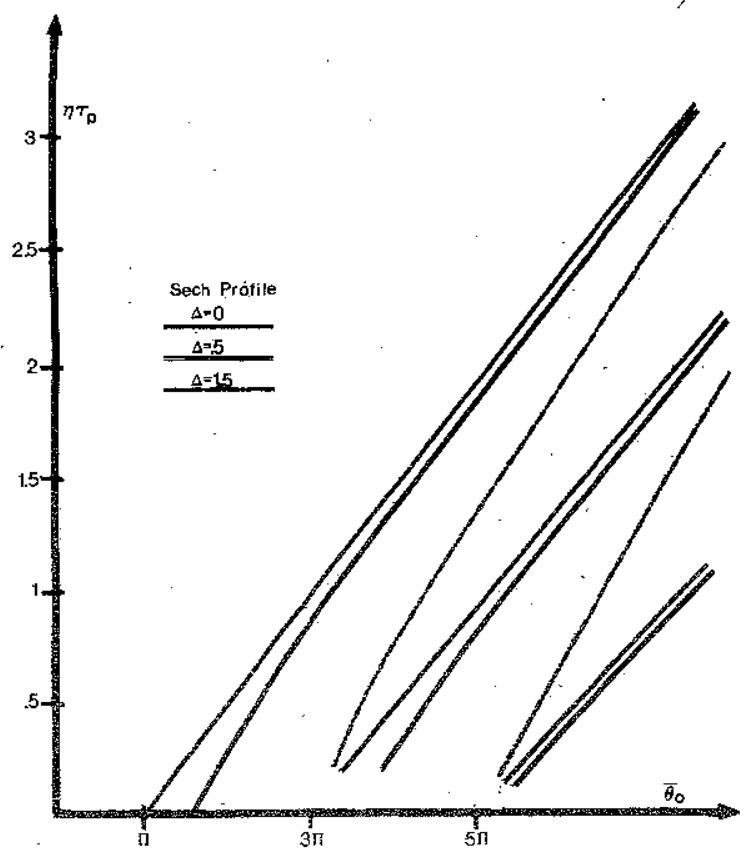

FIG. 2. Graph of $\eta \tau_{p}$ vs $\vec{\theta}_{0}$ for the sech profile with $\Delta=0,0.5,1.5$ and with $\beta=12$. 


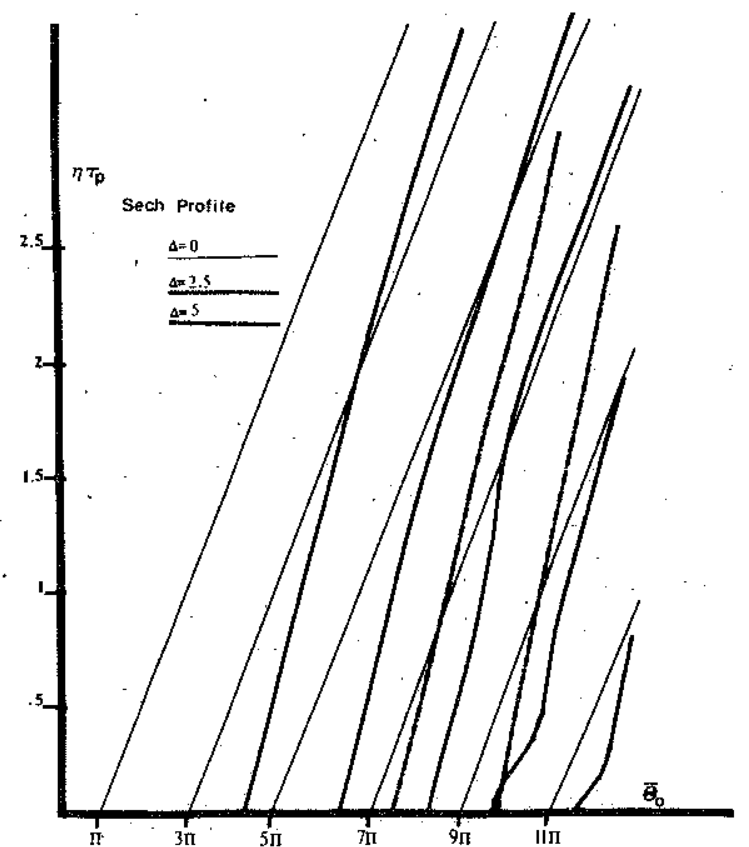

FIG. 3. Graph of $\eta \tau_{p}$ vs $\vec{\theta}_{0}$ for the sech profile with $\Delta=0,2,5,5$ and with $\beta=12$.

and

$$
\exp i \beta \Delta\left(\tau / \tau_{p}\right)^{2} /\left[\beta+\left(\tau / \tau_{p}\right)^{2}\right]
$$

produced almost identical results for $\beta$ large, we used the second form for more rapid calculations. In actuality, it was necessary to "clip". the sech and Gaussian profiles for numerical calculations, and they were clipped at $\pm 20 \tau_{p}$ for the sech and at $\pm 3 \tau_{p}$ for the Gaussian, since outside of this range, their relative magnitudes were less than $10^{-6}$. Then we arbitrarily choose $\dot{\beta}$ such that when $\tau \simeq 20 \tau_{p}$ for the sech and $\tau \simeq 3 \tau_{p}$ for the Gaussian, $\delta \simeq \tau / \tau_{p}$ when $\Delta \simeq 2$. So, we used $\beta=12$ for the sech and $\beta=3.12$ for the Gaussian.

In Figs. 2 and 3 we have plotted the results for the sech when $\Delta=0,0.5,1.5,2.5$, and 5 . In Fig. 4 we have similarly plotted the results for the Gaussian when $\Delta=0,5$, and 7 . In all cases, chirping causes all branches to shift to higher areas. This is contrary to the results for the box where only the first branch shifted to higher areas. Furthermore, in Fig. 2, we have not shown solutions for $\eta \tau_{p}$ very small. We found these values to be difficult to determine with any accuracy for our normal computer increments.

We therefore increased the accuracy by decreasing the step size in this portion of this graph. These results are shown in Fig. 5. Note that the fourth chirped branch is no longer straight and that it is shifted up to near the fifth unchirped branch. Also note that the values of $\eta \tau_{p}$ here are so small that all the unchirped branches now appear as straight lines. More surprising still are the fifth and sixth branches. Although the fifth branch does shift to higher areas when chirped, the sixth starts initially at lower areas when chirped but then quickly climbs into higher areas as. $\eta \tau_{p}$ gets large.

We have no ready explanation as to why these curves should vary so rapidly at low values of $\eta \tau_{p}$. However, we do note that at low values of $\eta \tau_{p}$, one does expect the far structure of the profile to affect these curves, and that this far structure was naturally absent in the box profile. Thus, these variations are undoubtedly related to how fast the profile is vanishing as $\tau \rightarrow \pm \infty$, and also to how strong the chirp is. For example, we have noted that for sufficiently large values of $\Delta$, these rapid variations at the small values of $\eta r_{p}$ always go away, possibly because of scaling to even smaller values of $\eta \tau_{p}$. We note that for the Gaussian, we only have calculations for $\Delta=$ 0 , 5 , and 7 , and that we did not then observe these rapid variations at low $\eta \tau_{p}$. It is possible that at $\Delta=5$ or 7 , these variations only occurred for values of $\eta \tau_{p}$ lower than what we looked for.

At the smaller values of $\Delta$, it was possible to check the numerical calculations against a perturbation calculation, as was done for the box profile. This we have done for the sech; and the trend is certainly not the same as was seen in Figs. 2 and 3 , where when $\Delta$ and $\eta \tau_{p}$ were both of order unity, all branches shifted to higher areas. The numerical results are shown in Fig. 6 for the first three branches, for $\Delta=0.05$ and 0.10 . Note that the first two branches still shift to higher areas, but the third one definitely shifts down. We have verified that this is indeed real by using the perturbation solution of the Zakharov-Shabat equation (see Appendix B). Again, letting $\gamma_{n}$ designate the shift in the chirped threshold from the unchirped threshold, we find, for the sech when $\Delta=0.1$, that

$$
\begin{aligned}
\gamma_{1} & =0.256 \\
\gamma_{2} & =0.011, \\
\gamma_{3} & =-0.093,
\end{aligned}
$$

which agrees very well with the numerical results.

Thus, the picture which is starting to emerge for chirped profiles like the sech and the Gaussian is as follows: For sufficiently high values of $\eta \tau_{p}$, all branches shift to higher areas. For a certain range of low $\eta \tau_{p}$ values depending on $\Delta$, we can expect a region of anomalous behavior. What happens here is not entirely clear, but apparently the higher branches

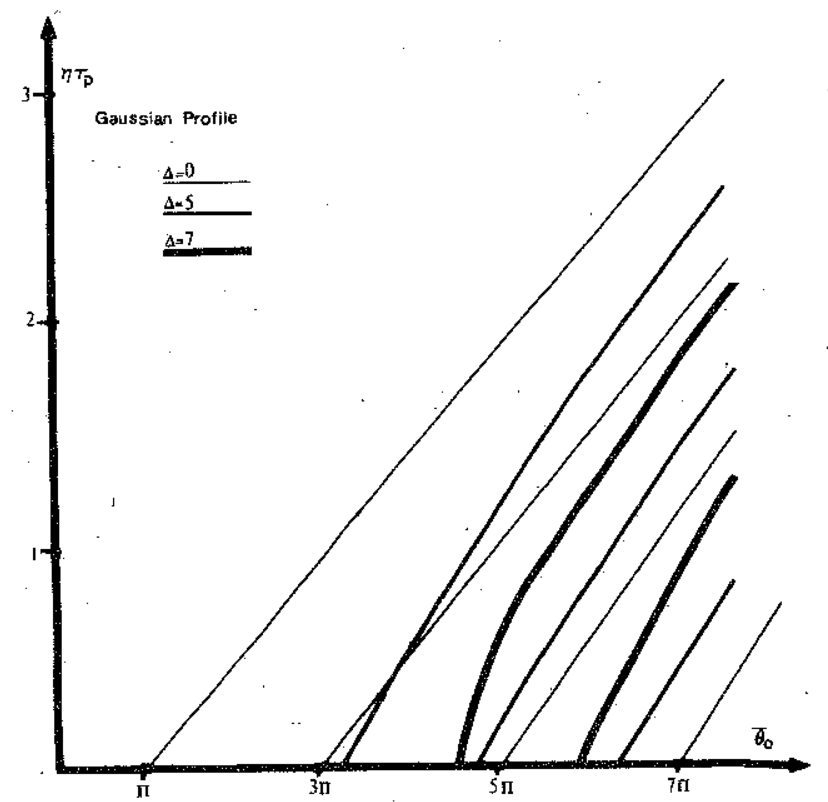

FIG, 4. Graph of $\eta \tau_{\rho}$ vs $\vec{\theta}_{0}$ for the Gaussian profile with $\Delta=0,5,7$ and with $\beta=3.12$. 
FIG. 5. Graph of $\dot{\eta} \tau_{p}$ vs $\bar{\theta}_{0}$ for the sech profile with $\eta$ small and computer precision increased. $\beta$ $=12$.

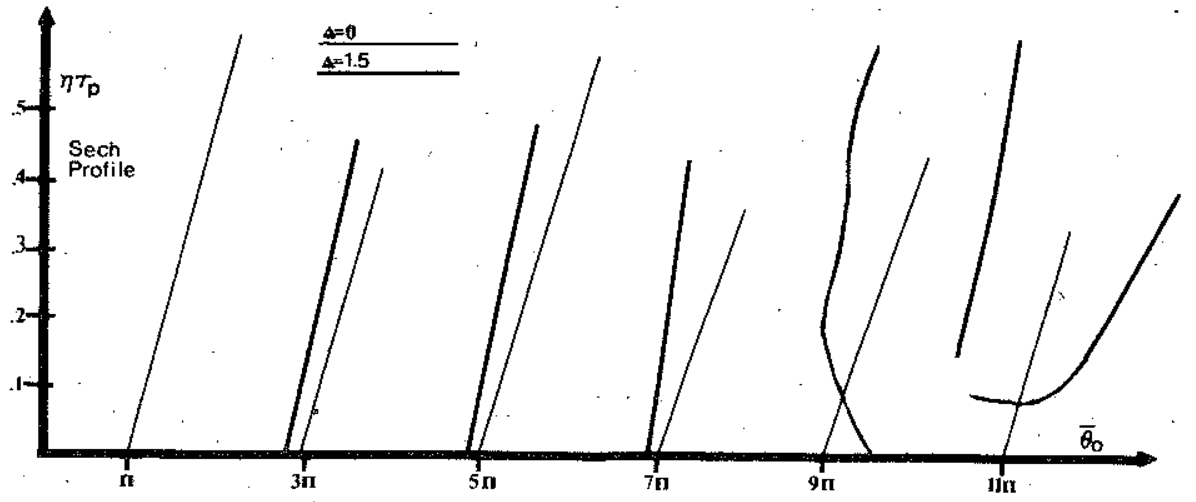

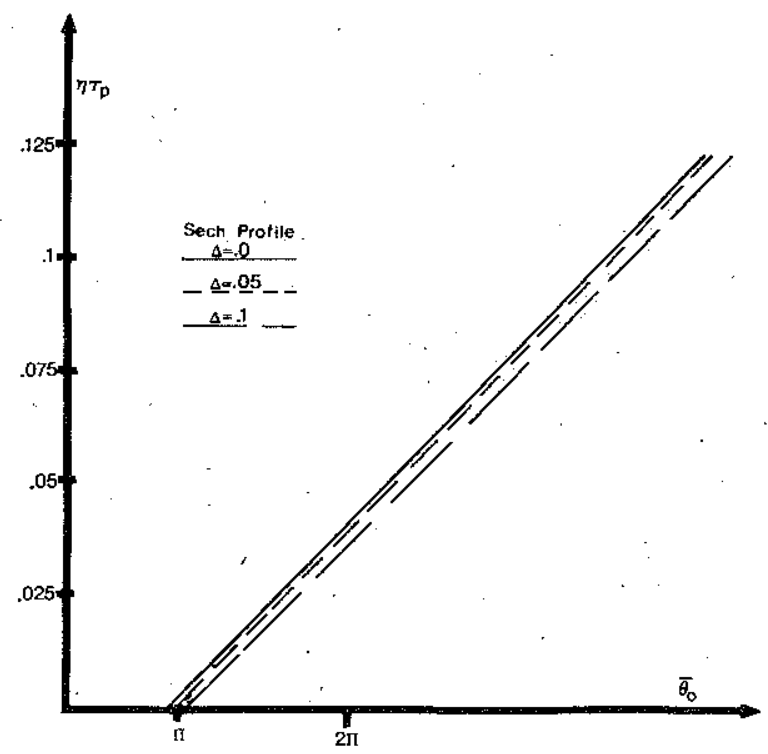

FIG. 6A. Graph of $\eta r_{p}$ vs $\bar{\theta}_{0}$ for the sech profile with $\Delta=0,0.05,0.1$, with $\eta$ very small, and with only the first branch plotted. $\beta=12$.

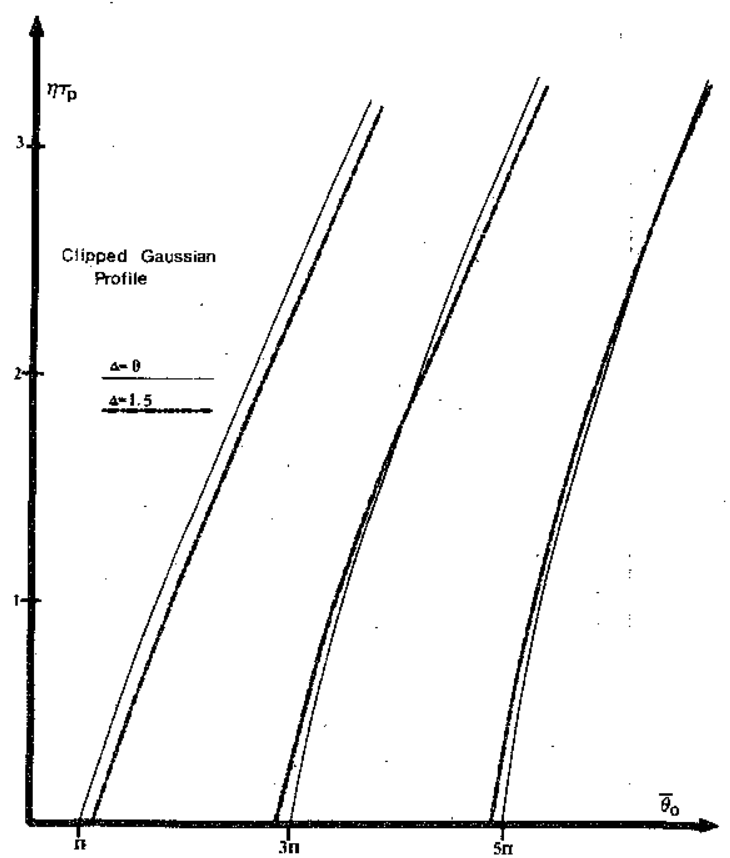

FIG. 6B. Graph of $\eta \tau_{\rho}$ vs $\vec{\theta}_{0}$ for the sech profile with $\Delta=0,0.05,0.1$, with $\eta$ very small, and with only the second branch plotted. $\beta=12$.

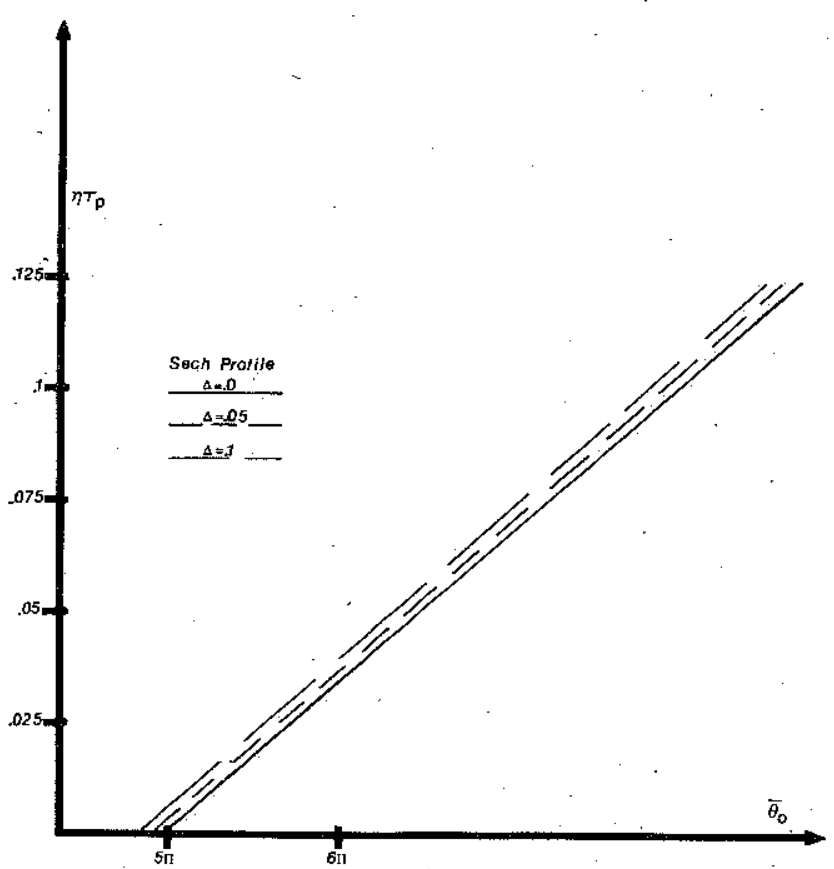

FiG. 6C. Graph of $\eta \tau_{p}$ vs $\bar{\theta}_{0}$ for the sech profile with $\Delta=0,0.05,0.1$, with $\eta$ very small, and with only the third branch plotted. $\beta=12$.

tend to extend into regions of profile area less than that for the unchirped case. Our results certainly indicate this, but more needs to be done before one can be more definite than this.

Lastly; we looked at what would happen if we strongly clipped either the sech or the Gaussian. The results are as one would expect from a simple WKB analysis, ${ }^{5}$ where the classical turning points are given approximately by $\kappa \epsilon= \pm 2 \eta$. Thus if the profile is clipped outside of the turning points $\left(\eta \tau_{p}\right.$ large), then the solution is not affected (significantly) by . clipping. But if the turning points are located where the profile is clipped ( $\eta \tau_{p}$ smaller), then profile appears to be more "box-like." This is shown in Fig. 7, where we have clipped the Gaussian profile at $\tau= \pm \tau_{p}$. Note that the second and third branches of the clipped Gaussian are "boxlike" at low $\eta \tau_{p}$ values, and as $\eta \tau_{p}$ increases, there is a smooth transition back to the "Gaussian-like" characteristics at higher $\eta \tau_{p}$ values.

As a concluding note concerning the sech pulse, we can compare our results for the chirped sech with those of Deck and Lamb, ${ }^{13}$ where they used the infinity of conservation laws 


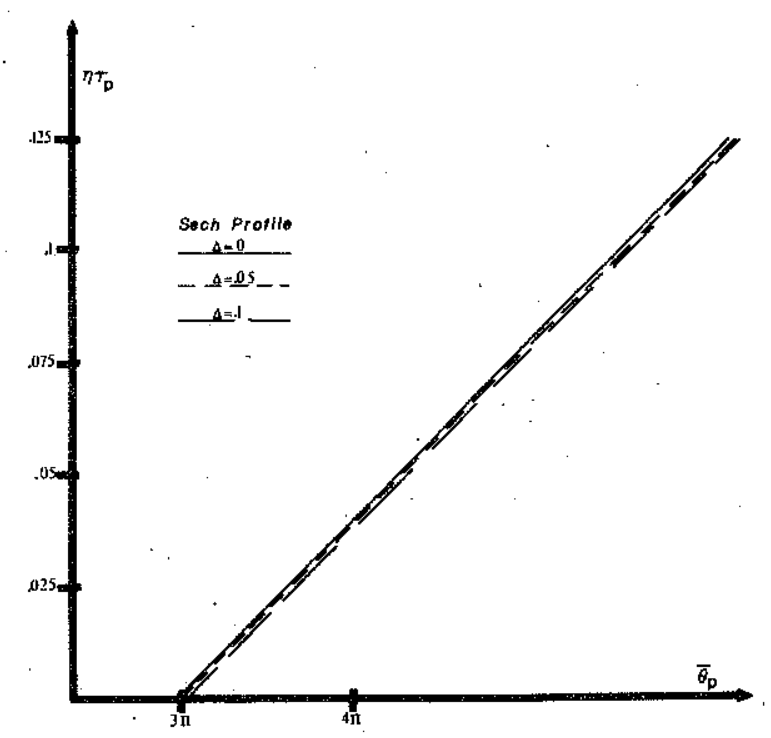

FIG. 7. Graph of $\eta \tau_{p}$ vs $\bar{\theta}_{0}$ for the "clipped" Gaussian profile with $\Delta=$ $0,1.5$ and with $\beta=3.12$.

to determine the "relative soliton pulse height," $\left(\eta / \bar{\theta}_{0}\right)$, versus the incident area, $\bar{\theta}_{0}$. Deck and Lamb's results versus ours are shown in Fig. 8 with $\Delta=0.1$. In the case of Deck and Lamb, their $\delta$ was $\Delta\left(\tau / \tau_{p}\right)^{2}$ rather than $\Delta \beta\left(\tau / \tau_{p}\right)^{2} /\left[\beta+\left(\tau / \tau_{p}\right)^{2}\right]$ as in our case. Before we discuss and compare these two results, it would be best if we would first make certain pertinent remarks concerning the method of the infinity of conservation laws. That method is an approximation, and indeed is an extremely good approximation, providing its basic assumptions are not violated. One of its basic assumptions is that the initial profile is compoosed of almost pure solitons $(2 \pi$ pulses) so that one may neglect, or at least roughly approximate, the "radiation"5 contained in the initial profile. Another way of saying the above, is that all of these locally conserved quantities are assumed to be globally conserved, or at least that their loss can be estimated (i.e., all of the initial profile energy, etc., is transmitted). Thus one important criterion for using the infinity of convservation laws is that there must be a very low fraction of "radiation" in the initial pulse profile, where this radiation ${ }^{5}$ is simply the continuous spectrum of the Zakharov-Shabat eigenvalue problem, Eq. (3), and is that part of the pulse which is absorbed within the first few Beer's lengths. Now, as a general rule, the amount of radiation in any given initial pulse profile is roughly proportional to the amount of oscillations, sudden and rapid changes, etc., in the profile. ${ }^{5}$ Thus a very slowly varying profile, if it has sufficient area, will have a minimum amount of radiation. In this case, the method of the infinity of conservation laws should give its best results, and indeed it does. ${ }^{15}$ Furthermore, at those values of $\bar{\theta}_{0}\left[\bar{\theta}_{0} \simeq(2 \eta-1) \pi\right]$ where this method has its worst errors, the amount of the radiation is a maximum locally. 5 on the other hand, when one chirps any initial pulse profile, one is automatically introducing oscillations, which by the above general rule, means that the amount of radiation in the initial profile is being increased. Now, since an assumption for this method of conservation laws is less valid, one would expect this method to give less accurate results. Indeed it does, as can be seen in Fig. 8 , where the results of our exact method are compared against those of Deck and Lamb. For the first branch, the two results diverge when $\left(4 \eta \pi / \bar{\theta}_{0}\right)$ is less than about 0.5. This is typical for the method of conservation laws, ${ }^{5}$ in that it gives its worst results for low $\eta$. But, when $\left(4 \eta \pi / \bar{\theta}_{0}\right)$ is greater than about 0.5 , the two results are in almost exact agreement, for both the chirped and the unchirped cases. This is an advantage of the method of conservation laws, in that at times it can give excellent results in spite of approximations made. But consider the second branch. Although Deck and Lamb only obtained results for low $\eta$, note the direction of the shift, which is opposite from our exact result. Naturally, it is reasonable to expect that if their results for the second branch were extended to higher values of $\eta$, one would still expect very close agreement for the unchirped cases. However, it is not possible to say definitely what would happen for the chirped case, especially for larger values of the chirp strength $(\Delta)$. Based on their uniform shift at low $\eta$, one would expect this shift to continue, in which case their result would be predicting a shift in the wrong direction. If so, one would then have to say that for accurate prediction of final $2 \pi$-pulse heights, the method of the conservation laws is accurate only for the first branch, and then only for $\left(4 \eta \pi / \theta_{0}\right)$ greater than about 0.5 . On the other hand, if one is only interested in rough estimates and good qualitative behavior as a function of $\bar{\theta}_{0}$, this method, which is fast, convenient, and easy to apply, would give quite good results. However the predicted shifts for the second and higher branches may well be in the wrong direction, although certainly of the correct order of magnitude.

\section{CONCLUDING REMARKS}

Our work has illustrated the usefulness of the ZakharovShabat equation in determining critical parameters for SIT and, in particular, for the case of the chirped input pulse. When the McCall-Hahn area theorem is not useful or valid, one may use this equation to determine critical parameters.

Furthermore, we have noted that chirping always causes the first branch to increase in absolute area when the initial pulse is chirped. This effect is independent of the initial pulse profile as well as the chirp strength.

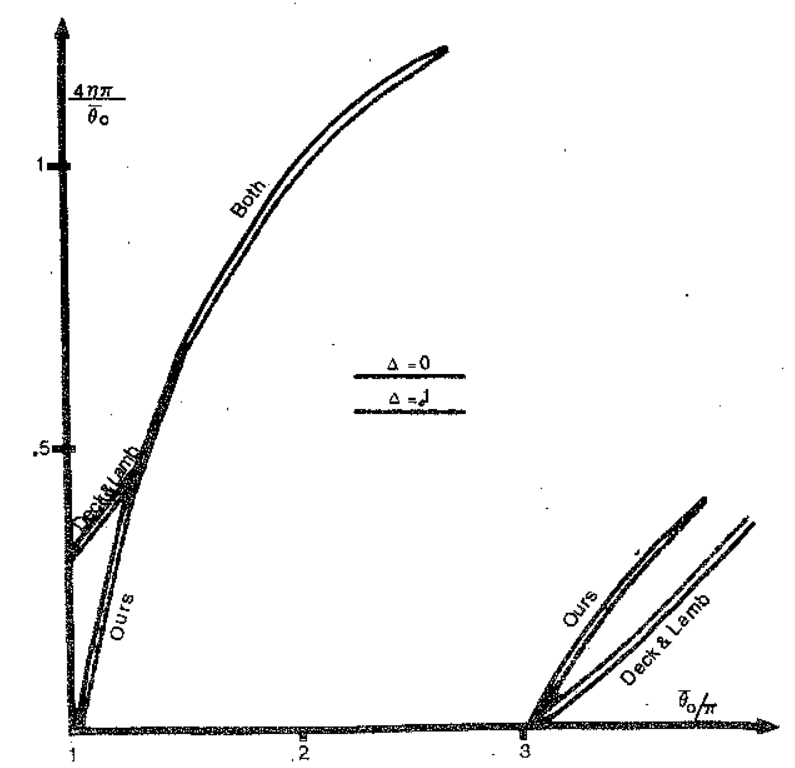

FIG. 8. The results of Deck and Lamb are graphed along with ours for $\Delta$ $=0,0.1$. 
The remaining higher branches may move up or down (in area) when chirped. This will depend both on the initial pulse shape and on the chirp strength. From our results, we can draw the following tentative conclusions: (i) For the box, chirping causes each upper branch to move down to lower areas. (ii) For a pulse with long tails (like the sech or Gaussian) chirping causes all branches (including the first one) to move up. However, for small to moderate values of $\Delta$, anomalous behavior can occur at very small values of $\eta$. (iii) For a pulse which is "clipped" and has its long tails cut off, each of these higher branches will tend to move down in area at small $\eta$ (like the box) and up in area at larger $\eta$ (like the Gaussian.)

\section{ACKNOWLEDGMENTS}

We wish to thank Alfred Scholldorf for writing the computer program we used to numerically solve the ZakharovShabat equation. This research was supported in part by NSF Grant No. MXS78-03979 and OWR Contract No. N00014-76-C-0867.

\section{APPENDIX A}

Location of bound-state eigenvalues for $\epsilon$ symmetric in $\tau$ Let us assume $\epsilon$ to be symmetric in $\tau$,

$$
\begin{gathered}
\epsilon(\tau)=\epsilon(-\tau), \\
v_{1 \tau}(\zeta, \tau)+i \dot{\zeta}_{1}(\zeta, \tau)=(1 / 2) \kappa \epsilon(\tau) v_{2}(\zeta, \tau), \\
v_{2 \tau}(\zeta, \tau)-i \zeta v_{2}(\zeta, \tau)=-(1 / 2) \kappa \epsilon^{*}(\tau) v_{1}(\zeta, \tau)
\end{gathered}
$$

In (A2) take $\zeta \rightarrow-\zeta$ and $\tau \rightarrow-\tau$, and also take the complex conjugate of the resulting equations. Then by using (A1), (A2) becomes

$$
\begin{aligned}
& v_{1 r}^{*}(-\zeta,-\tau)-i \zeta^{*} v_{1}^{*}(-\zeta,-\tau)=\frac{-\kappa \epsilon^{*}(\tau)}{2} v_{2}^{*}(-\zeta,-\tau) \\
& v_{2}^{*} \tau(-\zeta,-\tau)+i \zeta^{*} v_{2}^{*}(-\zeta,-\tau)=\frac{\kappa \epsilon(\tau)}{2} v_{1}^{*}(-\zeta,-\tau) .
\end{aligned}
$$

Thus if $\left[v_{1}(\zeta, \tau), v_{2}(\zeta, \tau)\right]$ is a solution of (A2), then $\left[v_{2}^{*}(-\zeta,-\tau)\right.$, $\left.v_{1}^{*}(-\zeta,-\tau)\right]$ is also a solution. Then from (A3) and (5) we have

$$
\phi(\zeta, \tau)=\left(\begin{array}{ll}
0 & 1 \\
1 & 0
\end{array}\right) \psi^{*}\left(-\zeta^{*},-\tau\right)
$$

Thus from (A4) it follows that

$$
a(\zeta)=a^{*}(-\zeta *)
$$

Therefore if $\zeta$ is a zero of $a(\zeta),-\zeta^{*}$ is also a zero of $a(\zeta)$, and if we have a bound-state solution corresponding to the eigenvalue $\zeta_{k}=i \eta_{k}+\xi_{k}$ where a $\left(\zeta_{k}\right)=0$, then we must also have another bound-state eigenvalue $-\xi_{k+1} *=i \eta_{k}+\xi_{k}=$ $i \eta_{k+1}-\xi_{k+1}$.

Thus even if $\epsilon$ is complex, but symmetric in $\tau$, bound-state solutions will occur either corresponding to $2 \pi$ pulses (for $\xi$ $=0$ ) or to breathers (for conjugately paired eigenvalues, $i \eta \pm$ $\xi)$.

\section{APPENDIX B}

Perturbation solution of the Zakharov-Shabal equation Consider the Zakharov-Shabat Eq. (3). If we define

$$
\begin{aligned}
& v_{1}=u_{1} e^{-i \zeta \tau}, \\
& v_{2}=u_{2} e^{+i \zeta \tau},
\end{aligned}
$$

then from (8)

$$
a=\left.u_{1}\right|_{\tau \rightarrow \infty}
$$

when we have $\left[u_{1}, u_{2}\right] \rightarrow[1,0]$ as $\tau \rightarrow-\infty$.

Now, let

$$
\begin{aligned}
& u_{1}=A(\tau) \cos Q / 2+B(\tau) \sin Q / 2, \\
& u_{2}=B(\tau) \cos Q / 2-A(\tau) \sin Q / 2,
\end{aligned}
$$

where $A, B$ are functions of $\tau$ which are to be determined and

$$
Q(\tau)=\kappa \int_{-\infty}^{\tau} \mathscr{E} d \tau
$$

Then, since bound-state solutions occur for $a=0$, we have at a zero of $a$, from (B2) and (B3) that

$$
-\left.(B / A)\right|_{\tau \rightarrow \infty}=\left.[\cot Q / 2]\right|_{\tau \rightarrow \infty}=\cot \left(\bar{\theta}_{0} / 2\right) .
$$

To find $\left.(B / A)\right|_{\infty}$, we simply use a perturbation expansion. (In some contexts, this is also referred to as the method of successive approximations.) From (B1), (B3), and (3), one can show that

$$
\begin{aligned}
(B / A)_{\tau}=\kappa \epsilon / 2\left\{(B / A)^{2}+1+(2 B / A) i \sin Q \sin (\delta+2 \zeta \tau)\right. & \\
- & {\left[(B / A)^{2}+1\right] \cos (\delta+2 \zeta \tau) } \\
+ & \left.i\left[1-(B / A)^{2}\right] \sin (\delta+2 \zeta \tau) \cos Q\right\}
\end{aligned}
$$

subject to $(B / A) \rightarrow 0$ as $r \rightarrow-\infty$.

For our work with the box and sech pulses we will simplify (B6) by taking $\zeta=i_{\eta}+\xi=0$ and expanding $\sin \delta$ and $\cos \delta$ in terms of $\Delta$. By making $\zeta$ zero, we are restricting attention to the critical or threshold areas (i.e., areas just large enough to produce one more soliton of zero pulse height). Also, since $(B / A)_{\tau}$, as $\tau \rightarrow \pm \infty$, drops off at least as fast as $\operatorname{sech}\left(\tau / \pi_{p}\right)$, we can approximate $\sin \delta$ and $\cos \delta$ by keeping only the first few orders in $\Delta$. Equation (B6) then becomes

$$
\begin{aligned}
(B / A)_{\tau}=\frac{1}{2} \bar{\theta}_{0}\left\{\left[(B / A)^{2}+1\right] \frac{\Delta^{2}\left(\tau / \tau_{p}\right)^{4}}{2}\right. \\
+(2 B / A) i \sin \left[\bar{\theta}_{0}\left(\tau / \tau_{p}+1 / 2\right)\right] \Delta \tau^{2} \\
\left.+\left[1-(B / A)^{2}\right] i \cos \left[\bar{\theta}_{0}\left(\tau / \tau_{p}+1 / 2\right)\right] \Delta \tau^{2}\right\}
\end{aligned}
$$

for the box.

First consider (B8). As a first trial solution, let $B / A(\tau \rightarrow$ $\infty)=B / A(\tau \rightarrow-\infty)=0$. Substitute this solution for $B / A$ into the right-hand side of (B8) and integrate the equation to find

$$
\begin{aligned}
(B / A)=\frac{\bar{\theta}_{0}}{2}\left[\frac{\Delta^{2}}{2} \int_{-1 / 2}^{\tau} d \tau \tau^{4}\right. & \\
& +i \int_{-1 / 2}^{\tau} d \tau \Delta \tau^{2} \cos \left[\bar{\theta}_{0}(\tau+1 / 2)\right],
\end{aligned}
$$

where we have set $\tau_{p}=1$ for convenience. Take (B9) and 
again substitute for $(B / A)$ into the right-hand side of (B8). Since the imaginary terms in (B8) duplicate the results pro- duced by the real terms, we can integrate (B8) and keep only the real second order terms in $\Delta$. Therefore, we get

$$
(B / A)=-\Delta^{2} \bar{\theta}_{0}^{2}\left[\sin \bar{\theta}_{0}\left(\frac{1}{2 \bar{\theta}_{0}^{4}}\right)+\cos \vec{\theta}_{0}\left(\frac{1}{\bar{\theta}_{0}^{5}}-\frac{1}{8 \bar{\theta}_{0}^{3}}\right)+\sin 2 \bar{\theta}_{0}\left(\frac{-49}{128 \bar{\theta}_{0}^{2}}+\frac{1}{32 \bar{\theta}_{0}^{6}}\right)+\cos ^{2} \bar{\theta}_{0}\left(\frac{-3}{16 \bar{\theta}_{0}^{3}}+\frac{11}{8 \bar{\theta}_{0}^{5}}\right)+\frac{1}{12 \bar{\theta}_{0}^{3}}-\frac{1}{\bar{\theta}_{0}^{5}}\right] .
$$

If we let $\bar{\theta}_{0}=(2 n-1) \pi+\gamma_{n}$ for $\gamma_{n}$. 1 then we have from (B10) and (B5)

$$
\gamma_{n}=\frac{\Delta^{2}}{(2 n-1)^{2} \pi^{2}}\left(\frac{2}{(2 n-1) \pi}-\frac{(2 n-1) \pi}{6}\right)
$$

Now consider the sech. Letting $\bar{\theta}_{0}=(2 n-1) \pi+\gamma_{n}$ and carrying out the perturbation expansion exactly as we did for the box, we get

$$
\left.\gamma_{n}=-4 \Delta^{2} \int_{-\infty}^{\infty} d \tau \frac{2 \beta \tau}{\left[\beta+\tau^{2}\right]^{2}} \sin \left[(2 n-1) \tan ^{-1} \sinh \tau\right)\right] \times \int_{-\infty}^{\tau} d \tau^{\prime} \frac{2 \beta \tau^{\prime}}{\left[\beta+\left(\tau^{\prime}\right)^{2}\right]^{2}} \cos \left[(2 n-1)\left(\tan ^{-1} \sinh \tau^{\prime}\right)\right] .
$$

We evaluated $\gamma_{n}$ in (B12) for $n=1,2,3$ numerically, and find that

$$
\begin{aligned}
& \gamma_{1}=0.2664 \\
& \gamma_{2}=0.01136 \\
& \gamma_{3}=-0.093
\end{aligned}
$$

1S. L. McCall and E. L. Hahn, Phys. Rev. Lett. 18, 908 (1967).

${ }^{2}$ S. L. McCall and E. L. Hahn, Phys. Rev. 183, 457 (1967).

3J. C. Diels and E. L. Hahn, Phys. Rev. 8, 1084 (1973).

${ }^{4}$ G. L. Lamb, Phys. Rev. Lett. 31, 196 (1973).

5D. J. Kaup, Phys. Rev. A 16, 704 (1977).

${ }^{6}$ M. J. Ablowitz, D. J. Kaup, and A. C. Newell, J. of Math Phys. 1852 (1974).

${ }^{7}$ R. E. Slusher and H. M. Gibbs, Phys. Rev. A 5, 1634 (1972).

${ }^{8}$ Gardner, Green, Kruskal, and Miura, Phys. Rev. Lett. 19, 1095 (1967).

${ }^{9}$ G. L. Lamb, Physica 66, 298 (1973).
${ }^{10}$ D. W. McLaughlin and J. Corones, Phys. Rev. A 10, 2051 (1974). 11V. E. Zakharov and A. B. Shabat, Soc. Phys. JETP 34, 62 (1972). 12M. J. Ablowitz, D. J. Kaup, A. C. Newell, and H. Segur, Stud. in App. Math. 53, 249 (1974).

${ }^{13}$ R. T. Deck and G. L. Lamb, Phys. Rev. A 12, 1503 (1975).

${ }^{14}$ D. F. Dubois, D. W. Forslund, and E. A. Williams, Phys. Rev. Lett. 33, 1013 (1974).

${ }^{15}$ D. D. Schnack and G. L. Lamb, Jr., in Coherence and Quantum Optics edited by L. Mandel \& E. Wolf. (Plenum, New York, 1973), p. 23-33. 